\title{
Clinical presentation of newly diagnosed diabetes patients in a rural district hospital in Eastern Uganda
}

\author{
Roy William Mayega ${ }^{1}$, Elizeus Rutebemberwa ${ }^{2}$
}

1. Department of Epidemiology and Biostatistics, School of Public Health, Makerere University College of Health Sciences

2. Department of Health Policy, Planning and Management, School of Public Health, Makerere University College of Health Sciences

\begin{abstract}
Background: Our objective was to describe the clinical presentation of new diabetes patients in a rural hospital, to enhance clinical detection in low resource settings.

Methods: A case series assessment of 103 new diabetes patients consecutively enrolled at Iganga Hospital in rural Eastern Uganda was conducted. All underwent a basic clinical assessment through the clinic's routine procedures.Following diagnosis, variables pertinent to the study (symptoms, blood pressure, anthropometry, and blood glucose) were secondarily abstracted from their clinical records.

Results: Fiftty two percent of new diabetes patients were female. The mean age was 49 years (SD=14.4). Two clinical symptoms were present in almost all new patients: Frequent urination (100\%) and frequent thirst (79\%). Moderately occurring symptoms (i.e. $25-50 \%$ of patients) included blurred vision, frequent eating and frequent sweating. The mean duration of symptoms was 1.4 years; $48 \%$ had high blood pressure while $46 \%$ were overweight. Random blood sugar was normal for $25 \%$ of patients. The majority $(71 \%)$ were classified as having 'moderate illness' at diagnosis. Severe illness was significantly lower among patients aged 40 or older compared to younger patients (OR 0.1; 95\% CI 0.03-0.35).

Conclusion: Out-patients aged 40-65 years should be prioritised for early diabetes diagnosis and associated risk factors in this setting.

Keywords: Diabetes, clinical presentation, newly diagnosed, unrecognized disease.

DOI: https://dx.doi.org/10.4314/ahs.v18i3.29

Cite as: Mayega RW, Rutebemberwa E. Clinical presentation of newly diagnosed diabetes patients in a rural district hospital in Eastern Uganda. Afri Health Sci. 2018;18(3): 707-719. bttps://dx.doi.org/10.4314/abs.v18i3.29
\end{abstract}

\section{Introduction}

The rising burden of type 2 diabetes in sub-Saharan Africa $^{1,2}$ calls for simplified approaches to identification of undetected cases at primary care facilities ${ }^{3}$. This requires

\section{Corresponding author:}

Roy William Mayega

Makerere University School of Public Health

P.O. Box 7072, Kampala, Uganda

Tel: +256-77-241-2455

E-mail: wromay2000@yahoo.co.uk a good understanding of how new patients present clinically in a context where more robust diagnostics are often inaccessible. According to the International Diabetes Federation, there were an estimated 10 million cases of diabetes in Africa and diabetes accounted for an estimated $6 \%$ of deaths on the continent in $2010^{4}$. The substantial advances in diagnosis, treatment and care for type 2 diabetes seen in high income countries are currently out of reach for many countries in sub-Saharan Africa ${ }^{5}$.

Diagnosis of type 2 diabetes is based on assessment of blood glucose levels using either the Oral Glucose Tolerance Test (OGTT), Fasting Plasma Glucose (FPG) or 
Glycated Haemoglobin (HbA1C). Diagnostic cut-offs for these tests have been defined by the World Health Organisation (WHO) and the American Diabetes Association (ADA $)^{6,7}$. In 2009, experts from the ADA, the European Association for the Study of Diabetes, and the International Diabetes Federation recommended that HbA1C should be the primary test for early detection of type 2 diabetes and pre-diabetes in asymptomatic persons ${ }^{6}$. However, due to resource constraints and late presentation, many health facilities in sub-Saharan Africa rely on random blood sugar (RBS),combined with explicit clinical symptoms for diagnosis of diabetes ${ }^{8}$. Reliance on Random Blood Sugar as a diagnostic test for diabetes in this setting implies that identifying asymptomatic diabetes is hardly practical ${ }^{8}$.

Because of the inadequate diagnostic capacity in primary care facilities, the rate of undiagnosed diabetes in sub-Saharan Africa is high ${ }^{9}$. In Uganda for example, 49\% of people with diabetes are unaware of their status ${ }^{10}$. Evidence shows that the majority of people with diabetes in low income countries present late, by which time they have irreversible complications ${ }^{3,11}$. However, our current knowledge of the clinical features of new diabetes patients presenting to primary care facilities is insufficient. One of the few studies that described the clinical presentation of newly diagnosed diabetes patients in Uganda showed that most patients presented with the classical symptoms of diabetes and severe hyperglycaemia ${ }^{12}$. However, that study was conducted at the National Referral Hospital and similar information is not available for rural patients. Rural clinics in sub-Saharan Africa rely substantially on overt clinical symptoms to identify people with diabetes ${ }^{11}$. In areas where more valid diagnostics are inaccessible, a clear understanding of the clinical profile of new patients can enhance the ability of first level health workers to identify suspicious cases, thereby reducing missed opportunities for detection.

Uganda's response to Non Communicable Disease (NCD) is only evolving ${ }^{13}$ and the majority of diabetes patients are managed at hospital level. Last revised in 2010, the Uganda Clinical Guidelines do not provide detailed guidance on early detection of type 2 diabetes ${ }^{14}$. Detailed guidelines for management of NCDs which were first developed in $1998^{15}$, have only been updated recently and have not been distributed to the districts.
The objective of this study was to describe the clinical presentation of newly diagnosed diabetes patients in a rural district hospital in Eastern Uganda so as to foster a better understanding of clinical features that can aid suspicion of unrecognized disease in settings where diagnostic facilities are lacking. This will facilitate the improvement of guidelines for passive case finding for undiagnosed diabetes in rural primary care facilities.

\section{Methods \\ Study site and context}

The study was conducted at Iganga District Hospital, located in Eastern Uganda, about 120 kilometers from Kampala, the capital city of Uganda. The district had an estimated population of 466,200 in $2013^{16}$, with public health centres, $83(82 \%)$ of which are government owned. Iganga Hospital is the only public hospital in the district, the rest being primary care facilities. The 100 bed hospital hosts the only diabetes clinic in the district, receiving patients from atleast five districts in the region. The diabetes clinic runs once a week on Tuesdays, and handles both type 1 and 2 diabetes. The clinic is run by a Clinical Officer (who undertakes the medical procedures including clinical assessments, requests for investigations, diagnosis and prescribing the requisite medications) and two nurses (who undertake basic physical assessments like anthropometry,administer the prescribed treatments, and provide health education). 'Clinical Officers' are a cadre of medical practitioners who in many countries in sub-Saharan Africa are equivalent to 'assistant doctors'. They are trained to handle minor out-patient medical and surgical tasks. However, because of severe shortages of qualified staff in Uganda's primary health care facilities, Clinical Officers often handle some specialised medical tasks that should be handled by Medical Officers, including diabetes care. The diabetes clinic at Iganga Hospital receives patients referred from the hospital's out-patients' department based on their symptoms at presentation. At the diabetes clinic, they are assessed and investigated by the attending clinical officer who then affirms the diagnosis. Those diagnosed with diabetes are recorded in a diabetes register and initiated on treatment by the Clinical Officer. All diabetes patients are required to keep a record book in which findings from their regular clinical evaluations are recorded by the attending clinical and nursing staff. 
The study population comprised all newly diagnosed diabetes patients (both type 1 and type 2) seen at Iganga Hospital's diabetes clinic from $1^{\text {st }}$ January 2013 to $30^{\text {th }}$ June 2014, a period of approximately 18 months. Approximately $90 \%$ of the population in Iganga resides in the rural areas with subsistence agriculture as the main economic activity. The main language in the region is ' $\mathrm{Lu}$ soga' a Bantu dialect.

\section{Study design}

A case series study design involving a prospective secondary review of clinical records of all incident diabetes patients (both type-1 and type-2) seen over the study period was conducted. The rationale for using incident cases was to enable description of patients' clinical characteristics at the time when they had just been diagnosed with diabetes. The minimum sample size required for determining the clinical characteristics of diabetes patients, at a confidence of level of $95 \%$, a precision of $6 \%$, an estimated prevalence of the most common clinical characteristic of $90 \%$ and $5 \%$ possible unusable records was $102 \mathrm{pa}-$ tients. Obtaining this number of incident cases required 18 months of data collection. The estimated sample size was only sufficient for describing clinical characteristics of diabetes patients in general but would not enable stratification by type of diabetes (type-1 or 2).

\section{Data collection procedures}

Only new patients with a final diagnosis of type-1 or type- 2 diabetes, affirmed by an entry into the hospital's diabetes register during the study period, were included in this study. Data collection involved a secondary review of clinical records. The clinicians' assessments and initial management of newly confirmed diabetes patients were reviewed to extract data on the variables pertinent to the study. The clinical records were reviewed at the specific visit when the diagnosis of diabetes was made, after the patient had received care. Therefore, the data collection team did not undertake any direct clinical assessment on participants but rather reviewed data already recorded by the attending clinicians. Patients clinical records were taken from two main sources: the patients' information entered into the diabetes clinic register as well as the patients' individual record books. At Iganga hospital, particulars of all diabetes patients are entered into a register that captures patient demographic characteristics. Addi- tional information regarding the blood glucose level at diagnosis, clinical presentation, laboratory test results, the initial diagnosis and the treatment given are recorded in a note book kept by the patient and presented at every clinic day. Data collection was limited to variables that are routinely assessed and recorded by the clinicians. A data abstraction tool was used to extract the patient information from both the patient register and the patient note books. The data abstraction tool was administered by two nurse research assistants.

For each patient, data on the following variables were abstracted from their clinical records: Demographic characteristics (age, sex, occupation, education level), family history of diabetes, main symptoms at presentation and when they started, anthropometry (height, weight), blood pressure (BP), laboratory investigations done and their outcomes (including blood glucose tests, chest x-rays, eye examination), and the initial management decision after diagnosis (initiation on either oral hypoglycaemic drugs or insulin as an out-patient, initiation on lifestyle measures alone or admission to treat an emergency complication).

Nurses and clinicians in the diabetes clinic routinely assess these characteristics for new patients during their clinical evaluation using the following approaches: Demographic characteristics are assessed through questions to the patient (about age, occupation and education level) and observation (sex). Family history of type 2 diabetes is also assessed through questions to the patient. It is based on the occurrence of diabetes in atleast one first degree relative (parent or sibling) or more than one second degree relative (grand-parent or cousin). Presenting symptoms are assessed through questions to the patient about their health problem. Height is routinely measured using an improvised height metre inscribed onto the wall of the clinic's examination room. It is measured with the patients standing up-right. Weight is routinely measured using a standard weighing scale. The weighing scale undergoes regular 'zero-correction' at the start of every clinic day. Blood pressure is routinely assessed using a standard aneroid manometer.

Blood glucose is assessed using simple hand-held glucometers of the brand 'Accu-Chek ${ }^{\circledR}$ ' (AVIVA). Although the 
meter uses capillary blood, the results provided are corrected to provide plasma glucose levels as per the International Federation of Clinical Chemistry and Laboratory Medicine (IFCC) guidelines ${ }^{17}$. Blood is drawn from a finger prick using a lancing device or regular syringe needle. It is placed on special applicators which are placed in the glucometer. The glucometer then provides a reading after about five to ten seconds. The Oral Glucose Tolerance Test was performed according to standard procedures i.e. the patient ate $100 \mathrm{mg}$ of commercial glucose, waited at the clinic, and thereafter had a blood glucose test after two hours. The type of diagnostic blood glucose tests that patients underwent varied according to 1) the clinicians' decisions guided by usual practice, 2) convenience to the patient and the patient's willingness to return for more valid tests and 3) the fact that un-confirmatory tests like random blood sugar (RBS) could be sufficient if the clinical signs are strongly pointing to diabetes. As such, the diagnostic procedures did not always conform to WHO recommendations.

The test used to confirm the diagnosis of diabetes for most patients $(93 / 103 ; 90 \%)$ was the random blood sugar test (Table 1). For the majority of patients, their diagnosis was confirmed on the basis of a single blood glucose test $(83.5 \%)$ including $73.8 \%$ confirmed on the basis of a single random blood sugar test and $8.7 \%$ confirmed with a single Fasting Plasma Glucose (FPG) test. Only 1 patient had an Oral Glucose Tolerance test (OGTT) (Table 1). Only $16.5 \%$ of patients underwent two different blood glucose tests (RBS and FPG). All patients who underwent two blood glucose tests were found to have initially tested negative with the RBS test, following which an FPG test was ordered. Among other tests conducted, about 10\% of patients had a chest $\mathrm{x}$-ray, while about $17 \%$ had an eye examination done (Table 1$)$.

Table 1: Tests undertaken on new diabetes patients

\begin{tabular}{|r|r|l|}
\hline Characteristic & No & \% \\
\hline Blood sugar tests conducted: & & \\
\hline Random Blood Sugar & 93 & 90.3 \\
\hline Fasting Plasma Glucose & 26 & 25.2 \\
\hline Urine sugar or other urine parameters & 17 & 16.5 \\
\hline Oral Glucose Tolerance Test & 1 & 1.0 \\
\hline Other tests conducted: & & \\
\hline Chest X-ray & 10 & 9.7 \\
\hline Full Haemogram/CBC & 1 & 1.0 \\
\hline Eye examination & 17 & 16.5 \\
\hline
\end{tabular}

Initial management decision referred to the initial treatment strategy that the clinician prescribed on the visit at which a diagnosis of diabetes was made, based on their assessment of the patients' severity of illness. The management options included: management as an out-patient either with lifestyle therapy only or with oral hypoglycaemics (classified as moderate illness at diagnosis) or management with insulin with or without initial admission (classified as severe illness at initial diagnosis). This information was abstracted from the patients' journals.
In the study context, management decisions for patients were taken by clinicians guided by the Uganda Clinical Guidelines ${ }^{14}$.

Respondents who reported having any first degree relative or more than one second degree relative with diabetes were classified as having a family history of type 2 diabetes. Duration since onset of illness was categorised into less than 1 year and $\geq 1$ year. Using the patients' anthropometric data,body mass index (BMI) was 
computed as weight (in kilograms) divided by the square of height (in metres). BMI was classified using standard WHO cut-offs: A BMI less than $18.5 \mathrm{Kgm}^{-2}$ was classified as underweight, that of 18.5-24.9 as normal, that of 25-29.9 $\mathrm{Kgm}^{2}{ }^{2}$ was classified as overweight while a BMI $\geq 30 \mathrm{Kgm}^{-2}$ was classified as obese ${ }^{18}$. Blood pressure was also classified using the standard World Health Organisation (WHO) cut-offs ${ }^{19}$. A participant was classified as having high blood pressure if his/her diastolic BP was $\geq 90 \mathrm{mmHg}$ or their systolic BP was $\geq 140 \mathrm{mmHg}$ or if they were already on anti-hypertension medications at the time of diabetes diagnosis. Classification of blood glucose was based on the standard cut-offs by the WHO. For Random Blood Sugar, a reading $\geq 11$ mmoll-1 was classified as diabetes. For Fasting Plasma Glucose, a cut off of $\geq 7 \mathrm{mmol}-1$ indicated diabetes while for the Oral Glucose Tolerance Test (OGTT), a cut off of $\geq 7.8$ mmoll-1 was classified as diabetes ${ }^{7}$.

Classification of type 1 or type 2 diabetes is a challenge in this setting. In the standard classification, type 1 diabetes is affirmed if tests show absolute deficiency of insulin while type- 2 diabetes is considered among patients who have high levels of exogenous insulin ${ }^{20}$. Because the diabetes clinic in our study setting has no capacity to determine insulin levels, their routine practice is to classify all patients aged 20 years or less as type 1 diabetes and all older patients as type- 2 diabetes. For this study's analysis however, a patient was presumed to have type 1 diabetes if he/she was aged less than 40 years, and also had a low or normal $\mathrm{BMI}^{20,21}$. All patients older than 40 years and those younger than 40 but with a high BMI were presumed to have type 2 diabetes $^{20,21}$. The 40 year cut-off was selected other than 35 or 30 years because the study district is a relatively low risk setting for type 2 diabetes $^{20}$.

\section{Statistical analysis}

Data was entered into SPSS Version 17 statistical software. The statistical analysis conducted was mainly descriptive. Frequencies were run for key background demographic characteristics and clinical features that were assessed in this study - findings were presented as frequencies and proportions. Cross-tabulations were conducted for demographic characteristics associated with severity of illness at initial diagnosis. Statistical differences between the comparison and indicator categories of the independent variables with the dependent variable (Severity of illness at diagnosis) were tested using Odds Ratios and p-values, at a significance level of $\alpha=0.05$. Comparisons that yielded statistically significant outcomes at bivariate analysis were subjected to logistic regression to control for possible confounding. Key findings from the analysis are presented in summary tables.

\section{Ethical considerations}

This study was presented to and approved by the Higher Degrees Research and Ethics Committee of Makerere University School of Public Health. The main ethical challenge encountered in implementation of the study was the shortage of diagnostic devices especially for blood glucose testing at the diabetes clinic. This was mitigated by supplying supplemental glucometers and testing kits to the clinic. The research team also ensured that the data abstraction tools did not capture the patients' names as a measure for confidentiality. Other ethical challenges arose from the finding that some diagnostic procedures for type 2 diabetes used in the clinic were not in line with recommendations from current international guidelines for diagnosis of the disease. However, these procedures are part of the usual standard of care in the study setting.

\section{Results \\ Demographic characteristics of newly diagnosed di- abetes patients}

The mean age of incident diabetes patients was 49 years $(\mathrm{SD}=14.4$ years; range 5 to 86 years). The median and modal age was 50 years, suggesting that the age distribution of newly diagnosed diabetes cases is normal. Based on the standard deviation, about two thirds of new diabetes patients $(68 \%)$ lie between the ages 35 to 63 years. About one quarter of incident diabetes cases are below the age of 43 years. Other demographic characteristics of newly diagnosed diabetes patients are summarized in Table 2. From the table, three quarters $(77 \%)$ of newly diagnosed diabetes patients were below the age of 60 years. The majority of newly diagnosed diabetes patients (58/103; 56\%) were aged between 40-49 years and 5059 years (Table 2). Two thirds of newly diagnosed diabetes patients were peasants/un-skilled workers. About two thirds had not had formal education beyond primary school. A family history of diabetes was reported in $20 \%$ of newly diagnosed diabetes patients (Table 2). A slight majority of newly diagnosed diabetes patients were females $(52 \%)$.

African Health Sciences Vol 18 Issue 3, September, 2018 
Table 2: Demographic characteristics of newly diagnosed diabetes patients

\begin{tabular}{|c|c|c|}
\hline Characteristic & No & $\%$ \\
\hline \multicolumn{3}{|l|}{ Age (In years): } \\
\hline$<30$ & 10 & 9.7 \\
\hline $30-39$ & 11 & 10.7 \\
\hline $40-49$ & 29 & 28.2 \\
\hline $50-59$ & 29 & 28.2 \\
\hline $60+$ & 24 & 23.3 \\
\hline \multicolumn{3}{|l|}{ Sex: } \\
\hline Male & 49 & $\overline{47.6}$ \\
\hline Female & 54 & 52.4 \\
\hline \multicolumn{3}{|l|}{ Occupation: } \\
\hline Subsistence farmer & 69 & 67.0 \\
\hline Trader/semi-skilled & 14 & 13.6 \\
\hline Civil servant/skilled & 20 & 19.4 \\
\hline \multicolumn{3}{|l|}{ Education level: } \\
\hline None & 21 & 20.4 \\
\hline Primary & 47 & 45.6 \\
\hline Secondary & 27 & 26.2 \\
\hline Tertiary & 8 & 7.8 \\
\hline \multicolumn{3}{|l|}{ Family history of diabetes: } \\
\hline $\begin{array}{llll} & \\
\end{array}$ & 21 & 20.4 \\
\hline No & 82 & 79.6 \\
\hline
\end{tabular}

\section{Clinical characteristics of new diabetes patients}

The most common symptom at presentation of new diabetes patients was frequent passing of urine. This symptom was reported by every patient assessed during the study period (Table 3). Frequent urination was followed by frequent drinking/thirst $(81 / 103$, i.e. $79 \%$ of all new patients) and general body weakness (52/103, i.e.51\%). Moderately occurring symptoms included: blurred vision (38\%), frequent eating (33\%), excessive sweating (27\%), joint pains $(22 \%)$, numbness $(21 \%)$ and headache $(21 \%)$ (Table 3). Forty-two percent of new diabetes patients reported a duration of less than 1 year since onset of symptoms. The mean time from onset of symptoms to diagnosis of diabetes was 1.4 years (median 1 year).

About one fifth of patients (19\%) were obese while slightly over one quarter $(26 \%)$ were overweight (Table 3$)$. Diabetes patients had a high prevalence of overweight and obesity: $45.6 \%$ of them (i.e. $47 / 103$ ) were overweight or obese while one fifth of the patients (20/103) were obese. It should be noted however that the majority $(45 / 103$ or $43.7 \%$ ) of new diabetes patients have a normal BMI. Additionally, 11 patients $(10.7 \%)$ were underweight. The mean BMI at presentation of new diabetes patients was 24.7 (median 24; range 13.3 - 44.6). About 48\% (49/103) of newly diagnosed diabetes patients had high blood pressure at presentation. 
Table 3: Presenting characteristics of new diabetes patients

\begin{tabular}{|c|c|c|}
\hline Characteristic & No & $\%$ \\
\hline \multicolumn{3}{|l|}{ Symptoms at presentation: } \\
\hline Frequent passing of urine & 103 & 100.0 \\
\hline Frequent drinking/thirst & 81 & 78.6 \\
\hline General body weakness & 52 & 50.5 \\
\hline Blurred vision & 39 & 37.9 \\
\hline Frequent eating/desire to eat & 34 & 33.0 \\
\hline Excessive sweating & 28 & 27.2 \\
\hline Joint pains & 23 & 22.3 \\
\hline Numbness of limbs & 22 & 21.4 \\
\hline Headache & 22 & 21.4 \\
\hline Loss of body weight & 13 & 12.6 \\
\hline Dizziness & 7 & 6.8 \\
\hline Body itching & 7 & 6.8 \\
\hline Loss of appetite & 6 & 5.8 \\
\hline Gaining of body weight & 6 & 5.8 \\
\hline General body pain & 5 & 4.9 \\
\hline Abdominal pains & 4 & 3.9 \\
\hline Blindness & 4 & 3.9 \\
\hline Hypertension & 3 & 2.9 \\
\hline Frequent urination, thirst and eating & 2 & 1.9 \\
\hline Fever & 2 & 1.9 \\
\hline Chest pain & 2 & 1.9 \\
\hline Numbness/Paraesthesias & 2 & 1.9 \\
\hline Nausea & 1 & 1.0 \\
\hline \multicolumn{3}{|l|}{ Duration since onset of symptoms: } \\
\hline$<1$ year & 43 & 41.7 \\
\hline$\geq 1$ year & 60 & 58.3 \\
\hline \multicolumn{3}{|l|}{ BMI at presentation: } \\
\hline Under-weight & 11 & 10.7 \\
\hline Normal & 45 & 43.7 \\
\hline Overweight & 27 & 26.2 \\
\hline Obese & 20 & 19.4 \\
\hline \multicolumn{3}{|l|}{ BP at presentation: } \\
\hline Normal & 54 & 52.4 \\
\hline Hypertensive & 49 & 47.6 \\
\hline \multicolumn{3}{|l|}{ Type of diabetes: $\uparrow$} \\
\hline Presumed to be type 1 diabetes & 14 & 13.6 \\
\hline Presumed to be type 2 diabetes & 89 & 86.4 \\
\hline \multicolumn{3}{|l|}{ Initial management track: +} \\
\hline \multicolumn{3}{|l|}{ Moderately ill } \\
\hline Outpatient on oral hypoglycaemics & 73 & 70.9 \\
\hline Outpatient on lifestyle therapy only & 1 & 1.0 \\
\hline \multicolumn{3}{|l|}{ Severely ill } \\
\hline Outpatient on insulin & 21 & 20.4 \\
\hline $\begin{array}{r}\text { Initially admitted; discharged on } \\
\text { insulin }\end{array}$ & 8 & 7.8 \\
\hline
\end{tabular}

$\dagger$ 'Presumed' to be type 1 diabetes if the patient was below 40 years of age with a normal or underweight BMI; 'Presumed' to be type 2 diabetes for all patients above 40 years, and those below 40 years but with a high BMI. This classification is considered presumptive because it was not possible to determine those who had absolute insulin deficiency vs. high exogenous insulin

$\ddagger$ Based on clinician’s assessment of severity of illness 
Regarding type of diabetes, $13.6 \%$ of patients fit the clinical criteria for presumptive classification as type 1 diabetes while $86.4 \%$ fit the criteria for type 2 diabetes. With regard to the initial management, the majority of patients were initially managed as out-patients on oral hypoglycaemic drugs $(71 \%)$ while one fifth of the patients $(20.4 \%)$ were initiated on insulin treatment as out-patients. About $8 \%$ of patients were initially admitted for treatment of emergency complications of diabetes. Only one patient $(1.0 \%)$ was managed with lifestyle measures only. Of 14 patients that fit the presumptive definition for type- 1 diabetes used for this study (i.e. aged less than 40 years with a normal or low BMI), 6 patients (42.9\%) were initially treated with oral hypoglycaemic drugs). However, 3 of the patients who fit the presumptive definition for type-1 diabetes were also found to be underweight, and all three were initiated on insulin.

\section{Distribution of blood glucose measurements}

The mean Random Blood Sugar level for 93 patients who underwent this test was 16.0 mmolL-1 (median 14.0; mode 24.7). However, the range was 5.4 - 37.0 mmolL-1 implying that some patients' random blood sugar levels were not in the range of diabetes. The mean FPG level for 26 patients who underwent the FPG test was 14.7
mmolL-1(median 12.7; mode 9.3; range 7.8 - 27.4) meaning that all patients who underwent the FPG test turned out with blood glucose levels in the range of diabetes. Of the 93 diabetes patients that underwent the Random Blood Sugar test, 25 (26.9\%) did not have blood sugar levels in the range of diabetes. Only 17 of these underwent a follow-on FPG test to affirm their diabetes status, meaning that 8 patients $(7.8 \%)$ were treated presumptively as having diabetes without confirmatory blood glucose testvalues showing their blood glucose level to be in the diabetes range.

\section{Demographic and clinical characteristics associated with initial management decision}

Adjusting for other demographic and clinical characteristics assessed in this study, the severity of illness at presentation was found to be significantly associated with two factors, both of which were demographic: age and occupation. Patients in the age-group 40-59 and those aged 60 years and older were $90 \%$ and $80 \%$ less likely to be severely ill at initial presentation compared to those aged less than 40 years (OR 0.1; 95\% CI 0.03-0.35 and OR 0.2 ; 95\% CI 0.04-0.64 respectively). Patients employed as skilled workers were $80 \%$ less likely to be severely ill at presentation compared to those whose occupation was peasants or unskilled workers (OR 0.2; 95\% CI 0.04-0.71). 
Table 4: Demographic and clinical characteristics associated with severity of hyperglycaemia at presentation

\begin{tabular}{|c|c|c|c|c|c|c|c|c|c|c|}
\hline \multirow[t]{2}{*}{ Characteristic } & \multicolumn{2}{|c|}{$\begin{array}{c}\text { Moderately ill at } \\
\text { presentation }\end{array}$} & \multicolumn{2}{|c|}{$\begin{array}{l}\text { Severely ill at } \\
\text { presentation }\end{array}$} & \multirow[t]{2}{*}{ COR } & \multirow[t]{2}{*}{ p-value } & \multirow[t]{2}{*}{$95 \% \mathrm{CI}$} & \multirow[t]{2}{*}{ AOR } & \multirow[t]{2}{*}{ p-value } & \multirow[t]{2}{*}{$95 \% \mathrm{CI}$} \\
\hline & No & $\%$ & No & $\%$ & & & & & & \\
\hline \multicolumn{11}{|l|}{ Age: } \\
\hline$<40$ & 8 & (38.1) & 13 & $(61.9)$ & 1.0 & & & 1.0 & & \\
\hline $40-59$ & 49 & $(84.5)$ & 9 & (15.5) & 0.1 & $<0.001^{* *}$ & $0.04-0.35$ & 0.1 & $>0.001$ & $0.03-0.35^{* *}$ \\
\hline $60+$ & 17 & $(70.8)$ & 7 & $(29.2)$ & 0.3 & $0.031^{* *}$ & $0.07-0.88$ & 0.2 & 0.011 & $0.04-0.64 * *$ \\
\hline \multicolumn{11}{|l|}{ Sex: } \\
\hline Male & 37 & $(75.5)$ & 12 & $(24.5)$ & 1.0 & & & 1.0 & & \\
\hline Female & 37 & $(68.5)$ & 17 & $(31.5)$ & 1.4 & 0.432 & $0.60-3.36$ & 1.4 & 0.566 & $0.47-4.04$ \\
\hline \multicolumn{11}{|l|}{ Occupation: } \\
\hline Unskilled & 45 & $(65.2)$ & 24 & $(34.8)$ & 1.0 & & & 1.0 & & \\
\hline Skilled & 29 & $(85.3)$ & 5 & (14.7) & 0.1 & $0.033^{* * *}$ & $0.11-0.94$ & 0.2 & 0.016 & $0.04-0.71^{* *}$ \\
\hline \multicolumn{11}{|l|}{ Education level: } \\
\hline Primary and below & 51 & $(75.0)$ & 17 & $(25.0)$ & 1.0 & & & 1.0 & & \\
\hline Post primary & 23 & $(65.7)$ & 12 & (34.3) & 1.6 & 0.321 & $0.64-3.80$ & 2.0 & 0.232 & $0.63-6.62$ \\
\hline \multicolumn{11}{|l|}{ Duration since onset: } \\
\hline$<1$ year & 27 & 62.8 & 16 & 37.2 & 1.0 & & & 1.0 & & \\
\hline$\geq 1$ year & 47 & 78.3 & 13 & 21.7 & 0.5 & $0.087^{*}$ & $0.20-1.17$ & 0.5 & 0.170 & $0.18-1.35$ \\
\hline \multicolumn{11}{|l|}{ BMI: } \\
\hline Normal & 39 & 69.6 & 17 & 30.4 & 1.0 & & & 1.0 & & \\
\hline Overweight/obese & 35 & 74.5 & 12 & 25.5 & 1.2 & 0.588 & $0.33-1.88$ & 1.1 & 0.906 & $0.37-3.05$ \\
\hline \multicolumn{11}{|l|}{ BP: } \\
\hline Normal & 38 & 70.4 & 16 & 29.6 & 1.0 & & & 1.0 & & \\
\hline Hypertensive & 36 & 73.5 & 13 & 26.5 & 0.9 & 0.727 & $0.36-2.03$ & 1.4 & 0.505 & $0.49-4.23$ \\
\hline
\end{tabular}

$\uparrow$ Initially treated with hypoglycaemic drugs or lifestyle measures

Initially treated with insulin or admission

\section{Discussion}

This study describes the clinical characteristics of newly diagnosed diabetes patients in a rural hospital in Eastern Uganda. The setting is a predominantly rural low income district. We show that three fifth of diabetes patients are aged between 40 and 59 years, and two thirds are within the age range of 35 to 65 years. Two clinical symptoms are present in almost all diabetes patients: Frequent urination and frequent drinking/thirst. The mean duration from onset of symptoms to diagnosis of diabetes was 1.4 years. The prevalence of concurrent non-communicable disease related risk factors for (hypertension and overweight) among diabetes patients was much higher than that in the general population. The majority of new patients were classified and hence treated as having 'moderate illness' at diagnosis.
Our study shows that the majority of new diabetes patients are aged between 40 and 59 years, with a mean age of 49 years. These rural patients are therefore on average older than those seen at Uganda's National Referral hospital, in whom the mean age was 45 years with incidence peaking between 40 to 49 years ${ }^{12}$. This is consistent with findings from a population-based survey of the prevalence of diabetes in the same setting which showed that diabetes increased consistently with age ${ }^{22}$. However, the age of patients in our study setting contrasts substantially with that from higher income countries where diabetes incidence is estimated to peak between 60 and 70 years $^{23}$. In our study, $75 \%$ of new diabetes cases were found to be aged below 60 years. These findings imply that diabetes is occurring in significantly younger age groups in our 
study setting, compared to higher income countries. The World Health Organisation estimates that about 30\% of NCD related deaths in LMICs occur in people aged less than 60 years, compared to $13 \%$ in the $\mathrm{HICs}^{24}$. This finding highlights the urgency to put in place measures for prevention and early detection of diabetes in rural low income settings.

Frequent urination and frequent thirst were found in almost all new diabetes patients. These symptoms are associated with substantially developed disease and they imply that by the time patients present with diabetes in the context where this study was conducted, their disease has progressed substantially. This is consistent with studies that show late presentation of diabetes in low income countries ${ }^{11}$. Nambuya and Otim also found substantial prevalence of classical symptoms and complications among newly diagnosed diabetes patients at Uganda's National Referral Hospital ${ }^{12}$. Late presentation in this setting is probably attributed to low patient awareness about diabetes and low clinical suspicion of diabetes by health workers. Recognition of diabetes is in many low income countries dependent on the presence of overt symptoms as current primary care services do not include diabetes screening for high risk individuals ${ }^{11}$. Since most patients first go to primary care facilities before going to hospitals, it is critical that lower level facilities are equipped with trained personnel and appropriate equipment to identify high risk persons for diabetes.

Even when symptoms have appeared, our study findings show that patients take an average of one-and-a-half years to get a diabetes diagnosis. This finding points to a health system setting (including first level health workers, diagnostic algorithms, and service delivery packages) that does not have sufficient capacity to detect early symptomatic cases. Patients therefore have to go through a long tortuous path before a definitive diagnosis of diabetes is made. Primary health care systems in this setting have for long been designed to address infectious diseases ${ }^{25}$, and would need significant re-tuning for chronic care ${ }^{26,27}$.

There is a call for simplification of diagnostic tools for diabetes $^{28,29}$. Lyon and colleagues have argued that in resource limited settings where point-of-care diagnostics are lacking, symptom-based tools may be the best hope for early identification of disease ${ }^{30}$. Since all diabetes sufferers in our study were found to have frequent passing of urine or frequent drinking/thirst, these symptoms can be useful pre-screening filters for possible early suspicion of undiagnosed diabetes cases at the lowest level of health facilities so that early referral for further investigations is prompted. Out-patients aged 35 to 65 years seeking care in first level health facilities could be passively asked about presence of these symptoms, their presence indicating a high likelihood of diabetes. Combinations of other signs and symptoms that were found to be common in diabetes patients (overweight or obesity, hypertension and body weakness) can also enhance the predictive value of these assessments.

This study found a high prevalence of concurrent cardiovascular risk factors among newly diagnosed diabetes patients. The prevalence of obesity was five times higher than its prevalence in the general population of Iganga district in a recent study in the same population ${ }^{31}$. Nambuya and Otim also found similar levels of obesity among new diabetes patients seen at the National Referral Hospital in Uganda ${ }^{12}$. The prevalence of both overweight and obesity in our study participants was 2.5 times higher than that found among people aged 35-60 years in a recent national level survey ${ }^{31}$. Likewise, the prevalence of hypertension in our study was twice that found among people aged 35-60 years in Iganga District ${ }^{31,32}$ and at the national leve ${ }^{10}$. Hypertension has been determined to be the most frequent concurrent cardiovascular risk factor among diabetes patients in Africa, ranging from 44-65\% prevalence in different studies ${ }^{8}$. In addition to the need to control blood glucose therefore, diabetes patients in this setting require multiple treatments and lifestyle education to address other risk factors like obesity and hypertension.

The majority of new diabetes patients were classified as having moderate illness at diagnosis, and were therefore managed with oral hypoglycaemic drugs. This finding is in contrast to studies that show that most people with type 2 diabetes present when the disease is severe ${ }^{5,11}$. However, the predominance of oral hypoglycaemic drugs as the initial treatment, even among patients who suit the clinical picture of type 1 diabetes, are associated with in- 
ability to assess insulin levels at diagnosis, difficulties in ensuring access to insulin, low levels of patient autonomy in self-management, challenges in management of the insulin cold chain, as well as inadequacies in the intense follow-up required for patients on insulin in this setting, which drive clinicians to initiate less patients on insulin than required.

The finding that one quarter of patients' random blood sugar levels were below the diabetes range strengthens the case for the ineffectiveness of random blood sugar as a screening test for diabetes. Current global guidelines for diabetes management discourage the use of RBS as a diagnostic test ${ }^{6,7}$, yet it is the most widely used test in Uganda. The finding that all patients tested with the FPG test returned positive results for diabetes may imply that FPG has a high diagnostic value for diabetes in this setting if combined with presence of symptoms. It is consistent with studies that have shown that both FPG and HbA1C have a relatively high accuracy at current cut-offs ${ }^{33}$.

The main limitations of this study arose from the study context. In this rural low resource setting, the only diabetes clinic in the districts relies majorly on random blood glucose for confirmation of diabetes. However, combination of the RBS with clinical signs, and the late presentation of patients in settings such as these $\mathrm{e}^{3,11}$ increases the predictive value of the RBS test. In the study setting, blood glucose tests are conducted using pointof-care rapid test kits, which might affect accuracy of the results. However, there is evidence that modern point-ofcare tests have sufficiently high levels of accuracy ${ }^{34,35}$. The existence of patients treated without evidence of a high blood sugar also raises concerns, but affirms the need for capacity building in diabetes care. The absence of tests to assess insulin levels or autoimmune reactions meant that it was not possible to have accurate classification of type 1 and type 2 diabetes. This was partly mitigated by using practical clinical criteria for classification, whose limitation is acknowledged. The rate of patient flow affected the sample size, limiting the study utility to mainly descriptive purposes. The setting of the study being a hospital meant that the findings are mainly generalizable to hospital settings. However, this being the only diabetes clinic in the district meant that patients were drawn from the entire district.
In conclusion, new diabetes patients in this predominantly rural subsistence-based setting are on average substantially younger than those in higher income countries. The majority of patients present with classical symptoms of diabetes, showing that the disease is fairly advanced at diagnosis. The patterns of resort and the system failures that these patients experience before diagnosis should be investigated. There is a need for increased skills-building for first line health workers in rural hospitals to increase their acumen in clinical suspicion of diabetes, and a need for enhanced clinical algorithms to support risk assessment and early diagnosis of diabetes. Because two clinical symptoms are present in almost all diabetes patients (frequent urination and frequent drinking/thirst), and because most new patients are aged between 40-59, all adults within this age-group presenting to the out-patient department should be asked whether they have these symptoms, and investigated with a presumptive blood glucose measurement if they report to have them. All diabetes patients in this setting need to be assessed and appropriately managed for hypertension and obesity.

\section{Key messages}

- In this rural low income setting in Eastern Uganda, twothirds of new diabetes patients are aged between 35 to 65 years. This age-group should be the target of efforts at early detection, more especially those aged 40-59, where three fifths of new patients lie.

- Because two clinical symptoms are present in almost all diabetes patients (frequent urination and frequent drinking/thirst), all clients aged 40-59 presenting to the outpatient departments should be asked whether they have these symptoms.

- $50 \%$ of newly diagnosed diabetes patients had cardio-vascular or metabolic co-morbidities. All diabetes patients should be assessed for overweight and hypertension, which should also be prioritised in their treatment plan.

- Though widely used as a diagnostic tool in this setting, random blood sugar is not effective as it would misclassify about one quarter of patients as having a normal blood glucose.

\section{Conflict of interest}

The authors declare no conflict of interest.

\section{Acknowledgements}

Author contributions: ER conceptualized the study. 
RWM developed the study methodology, set up the retrospective study, trained the research assistants and collected the data. RWM and made the first draft of the manuscript. The manuscript draft was jointly edited and improved by both ER and RWM.

This work was primarily funded by the Swedish International Development Cooperation Agency through the Directorate of Research and Graduate Studies at Makerere University. The Management of Iganga District Hospital and its diabetes clinic especially Dr. Lubega Mohammed and Mr. Mpata Zaid are duly acknowledged for their technical input.

\section{References}

1. Mbanya JC, Motala AA, Sobngwi E, Assah FK, Enoru ST: Diabetes in sub-Saharan Africa. Lancet. 2010, 375(9733):2254-2266.

2. Maher D, Sekajugo J: Research on health transition in Africa: time for action. Health Res Policy Syst. 2011, 9:5.

3. Kengne A, Sobngwi E, Echouffo-Tcheugui J, Mbanya JC: New insights on diabetes mellitus and obesity in Africa-Part 2: prevention, screening and economic burden. Heart. 2013, 99(15):1072-1077.

4. Roglic G, Unwin N: Mortality attributable to diabetes: estimates for the year 2010. Diabetes research and clinical practice. 2010, 87(1):15-19.

5. Kengne AP, Echouffo-Tcheugui JB, Sobngwi E, Mbanya JC: New insights on diabetes mellitus and obesity in Africa-part 1: prevalence, pathogenesis and comorbidities. Heart. 2013a, 99(14):979-983.

6. American Diabetes Association: Standards of medical care in diabetes-2013. Diabetes Care. 2013, 36(Suppl 1):S11-66. doi: 10.2337/dc2313-S2011.

7. World Health Organization, International Diabetes Federation: Definition and diagnosis of diabetes mellitus and intermediate hyperglycemia: report of a WHO/IDF consultation. In.; 2006.

8. Kengne AP, Sobngwi E, Echouffo-Tcheugui JB, Mbanya JC: New insights on diabetes mellitus and obesity in Africa-Part 2: prevention, screening and economic burden. Heart. 2013b, 99(15):1072-1077.

9. Mbanya JC, Motala AA, Sobngwi E, Assah FK, Enoru ST: Diabetes in sub-Saharan Africa. Lancet. 2010, 375(9733):2254-2266.

10. Bahendeka S, Wesonga R, Mutungi G, Muwonge J, Neema S, Guwatudde D: Prevalence and correlates of diabetes mellitus in Uganda: a population-based national survey. Tropical Medicine \& International Health : TM \& IH. 2016, 21(3):405-416.

11. Ambady R, Chamukuttan S: Early diagnosis and prevention of diabetes in developing countries. Rev Endocr Metab Disord. 2008, 9(3):193-201. Epub 2008 Jul 2007.

12. Nambuya AP, Otim MA, Whitehead H, Mulvany D, Kennedy R, Hadden DR: The presentation of newly-diagnosed diabetic patients in Uganda. QJM : monthly Journal of the Association of Physicians. 1996, 89(9):705-711.

13. Ministry of Health: Health Sector Strategic Plan III 2010/11-2014/15. In. Kampala: Ministry of Health, Uganda; 2010a.

14. Ministry of Health: Uganda Clinical Guidelines: Guidelines on management of common conditions. In.; 2010b.

15. Masaba J, Baingana S, Odiit A, Nalukenge K, Ofubo L: Guidelines for non-communicable diseases at District level In. Official Website of the Ministry of Health, Republic of Uganda: Ministry of Health, Uganda; 1998.

16. Uganda Bureau of Statistics: Projections of demographic trends in Uganda 2007-2017. In., vol. 1: Uganda Bureau of Statistics 2007.

17. ROCHE: Accu-Chek ${ }^{\circledR}$ Aviva test strips provide accurate and reliable blood glucose measurements. In: Evaluation Report: Accu-Chek ${ }^{\circledR}$ Aviva Test Strips with Advanced Chemistry. ROCHE; 2013.

18. World Health Organization: Physial Status: The Use and Interpretation of Anthropometry: Report of a WHO Expert Committee. In. Edited by WHO; 1995.

19. Whitworth JA: 2003 World Health Organization (WHO)/International Society of Hypertension (ISH) statement on management of hypertension. $J$ Hypertens. 2003, 21(11):1983-1992.

20. Hope SV, Wienand-Barnett S, Shepherd M, King SM, Fox C, Khunti K, Oram RA, Knight BA, Hattersley AT, Jones AG et al: Practical Classification Guidelines for Diabetes in patients treated with insulin: a cross-sectional study of the accuracy of diabetes diagnosis. The British journal of general practice: The Journal of the Royal College of General Practitioners. 2016, 66(646):e315-322.

21. Shields BM, Peters JL, Cooper C, Lowe J, Knight BA, Powell RJ, Jones A, Hyde CJ, Hattersley AT: Can clinical features be used to differentiate type 1 from type 2 diabetes? A systematic review of the literature. BMJ Open. 2015, 5(11):e009088.

22. Mayega RW, Guwatudde D, Makumbi F, Nakwagala 
FN, Peterson S, Tomson G, Ostenson CG: Diabetes and pre-diabetes among persons aged 35 to 60 years in eastern Uganda: prevalence and associated factors. PLoS One. 2013, 8(8):e72554.

23. Unwin N, Alberti KG: Chronic non-communicable diseases. Ann Trop Med Parasitol. 2006, 100(5-6):455-464.

24. World Health Organization: Global status report on noncommunicable diseases 2010: Description of the global burden of NCDs, their risk factors and determinants. In. Edited by WHO; 2011c.

25. Maher D, Smeeth L, Sekajugo J: Health transition in Africa: practical policy proposals for primary care. Bull World Health Organ. 2010, 88(12):943-948. Epub. 2010 Nov 2014.

26. Beaglehole R, Epping-Jordan J, Patel V, Chopra M, Ebrahim S, Kidd M, Haines A: Improving the prevention and management of chronic disease in low-income and middle-income countries: a priority for primary health care. Lancet. 2008, 372(9642):940-949. doi: 910.1016/ S0140-6736(1008)61404-X.

27. Bischoff A, Ekoe T, Perone N, Slama S, Loutan L: Chronic disease management in Sub-Saharan Africa: whose business is it? International Journal of Environmental Research and Public Health. 2009, 6(8):2258-2270.

28. Little RR: Analysis: point-of-care testing for glycated hemoglobin (GHB). Diabetes Technol Ther. 2005, 7(6):913915.

29. Mostafa SA, Davies MJ, Webb D, Gray LJ, Srinivasan BT, Jarvis J, Khunti K: The potential impact of using glycated haemoglobin as the preferred diagnostic tool for detecting Type 2 diabetes mellitus. Diabet Med. 2010, 27(7):762-769.

30. Lyon AW, Larsen ET, Edwards AL: The impact of new guidelines for glucose tolerance testing on clinical practice and laboratory services. Cmaj. 2004, 171(9):10671069.

31. Mayega RW, Makumbi F, Rutebemberwa E, Peterson S, Ostenson CG, Tomson G, Guwatudde D: Modifiable socio-behavioural factors associated with overweight and hypertension among persons aged 35 to 60 years in eastern Uganda. PLoS One. 2012, 7(10):e47632.

32. Wamala JF, Karyabakabo Z, Ndungutse D, Guwatudde D: Prevalence factors associated with hypertension in Rukungiri district, Uganda--a community-based study. Afr Health Sci. 2009, 9(3):153-160.

33. Shibata K, Suzuki S, Sato J, Ohsawa I, Goto S, Iritani I, S T: Diagnostic accuracy of glycohemoglobin A1c (HbA1c) for postprandial hyperglycemia was equivalent to that of fasting blood glucose. Journal of Clinical Epidemiology 2005, Volume: 58( Issue: 10):Pages: 1052-1057.

34. Shibata K, Suzuki S, Sato J, Ohsawa I, Goto S, Iritani I, S T: Diagnostic accuracy of glycohemoglobin A1c (HbA1c) for postprandial hyperglycemia was equivalent to that of fasting blood glucose. Journal of Clinical Epidemiology. 2005, 58(10):1052-1057.

35. Wood JR, Kaminski BM, Kollman C, Beck RW, Hall CA, Yun JP, Cengiz E, Haller MJ, Hassan K, Klingensmith GJ et al: Accuracy and precision of the Axis-Shield Afinion hemoglobin A1c measurement device. J Diabetes Sci Technol. 2012, 6(2):380-386. 